\title{
Metas de glucemia en la Diabetes tipo II
}

\author{
Glycemic Targets in Type 2 Diabetes. Letter, issue 68, January - February 2008
}

Traducido con autorización de "Therapeutics Initiative" (The University of British Columbia). Disponible en URL: http://www.ti.ubc.ca/PDF/68.pdf

Muchas personas con diabetes mellitus tipo 2 gastan mucho tiempo, esfuerzo y dinero tratando de mantener sus niveles de glucemia normales o cercanos a la normalidad.

¿Existe evidencia sólida para este enfoque? Accedimos a las guías canadienses publicadas en el sitio web de la Asociación Canadiense de Diabetes para averiguarlo'. Si bien las guías combinan recomendaciones para diabetes tipo 1 y tipo 2, para esta nota terapéutica hemos suprimido la información referida a la diabetes tipo 1.

1. Los objetivos de glucemia deben ser individualizados, sin embargo, el tratamiento en la mayoría de los pacientes con diabetes tipo 2 debería estar dirigido a lograr una hemoglobina A1C menor a $7 \%$ para reducir el riesgo de complicaciones microvasculares (Grado A, Nivel $1 \mathrm{~A})^{2}$, y complicaciones macrovasculares (Grado C, Nivel 3$)^{3}$.

2. Para lograr una hemoglobina A1C menor a $7 \%$, los pacientes con diabetes tipo 2 deberían tratar de lograr una glucemia plasmática en ayunas (GPA) o una glucemia plasmática preprandial (GPP) entre 72 y $126 \mathrm{mg} / \mathrm{dl}$ y una glucemia postprandial (GPP) a las dos horas entre 90 y $180 \mathrm{mg} / \mathrm{dL}$ (Grado B, Nivel 2) $)^{2,3}$.

3. Si puede lograrse de manera segura, se debe tratar de disminuir los objetivos de glucemia plasmática hacia el rango normal (Grado C, Nivel 3) 3,4:

- Hemoglobina A1C menor a 6\% (Grado D, Consenso).

- GPA / GPP: 72 a 108 mg/dL. (Grado D, Consenso).

- GPP a las dos horas: 90 a 144 mg/dL (Grado D, Consenso).

\section{¿Qué significan estas guías para los médicos?}

Las guías advierten que estos objetivos deben ser individualizados y no serán apropiados para todos los pacientes. Al mismo tiempo, abogan explícitamente para que estos objetivos sean aplicados a la mayoría. Las guías asumen que estos objetivos reducirán las complicaciones asociadas con la diabetes y que el beneficio de este enfoque es superior al daño.

Los objetivos crean graves problemas para los médicos y para los pacientes, que tienen importantes implicancias en términos de tiempo, esfuerzo y utilización de los recursos de la atención de la salud.

\section{¿Cuál es la evidencia utilizada para apoyar este enfoque?}

Grado A, Nivel 1 significa que se basa en una revisión sistemática de ensayos clínicos controlados aleatorizados de alta calidad, diseñados adecuadamente con el poder suficiente para responder a la pregunta planteada por los investigadores.

En este caso, la referencia utilizada para apoyar un objetivo de hemoglobina A1C de menor a 7\% es el estudio UKPDS332. Este ensayo aleatorizó 3.687 nuevos pacientes con diabetes tipo 2 al tratamiento con fármacos orales (sulfonilureas) o insulina, para lograr una meta de glucosa plasmática en ayunas menor a $108 \mathrm{mg} / \mathrm{dL}$, y lo comparó con el tratamiento con dieta sola para lograr las mejores glucemias plasmáticas en ayunas posibles.

Los pacientes fueron seguidos durante diez años y el promedio de los niveles de hemoglobina $\mathrm{A} 1 \mathrm{C}$ alcanzados en el grupo de fármacos y en el de dieta sola fueron $7 \%$ y $7,9 \%$, respectivamente.

Los puntos finales microvasculares se redujeron en el grupo de fármacos en comparación con la dieta sola (RRA 2,4\% [IC95\%
0,4 a 4,7\%] NNT 42 a diez años). Esta reducción se explica exclusivamente por la disminución de sólo uno de los puntos finales microvasculares -la necesidad de fotocoagulación retiniana, con un RRA $2,7 \%$. Sin embargo, la mortalidad total y los resultados macrovasculares no se redujeron y sí aumentaron los eventos mayores de hipoglucemia. Ver nota terapéutica Nro 27$)^{5}$.

Grado C, nivel 3 significa que se basa en evidencia proveniente de ensayos no aleatorizados, en este caso se hace referencia a una publicación de datos observacionales proveniente del UKPDS que había relacionado puntos finales con niveles de glucemia ${ }^{3}$.

Las recomendaciones Grado $D$ se basan en un consenso de los expertos que participaron en el informe.

\section{Asunciones de las guías}

Las guías mencionadas previamente asumen lo siguiente:

1. Que los médicos pueden identificar a los pacientes para quienes las metas de hemoglobina $\mathrm{A} 1 \mathrm{C}$ menores a $6 \%$ ó a $7 \%$ son seguras.

2. Que los beneficios superan el daño, cuando se trata a los pacientes identificados para lograr las metas de hemoglobina A1C de menores a $6 \%$ ó a $7 \%$, comparadas con metas superiores de hemoglobina $\mathrm{A} 1 \mathrm{C}$

Antes de febrero de 2008 no había pruebas para apoyar estos supuestos y desde febrero de 2008 , existe evidencia que pone en duda estas hipótesis.

\section{ACCORD, una prueba de la hipótesis sobre las metas de glucemia}

El ensayo clínico de acción para el control de riesgo cardiovascular en la diabetes (ACCORD) se diseñó para evaluar si la reducción de los niveles de hemoglobina A1C a un objetivo de menos de $6 \%$ en los pacientes diabéticos tipo 2 con alto riesgo vascular, podía reducir las enfermedades cardiovasculares $(\mathrm{ECV})^{6}$.

En este ensayo clínico 10.251 pacientes fueron aleatorizados a una meta de hemoglobina A1C menor a $6 \%$ o a una meta de 7 a 7,9\%. Otros componentes del mismo estudio fueron la asignación aleatoria de los pacientes a metas de presión arterial sistólica menores a $120 \mathrm{mmHg}$ ó menores a $140 \mathrm{mmHg}$; y a una estatina asociada a un fibrato o una estatina sola. La fecha prevista de finalización del ensayo fue 2009.

Luego de cuatro años de iniciado el estudio, en febrero de 2008, el componente de la glucemia se dió por terminado debido a que la mortalidad fue del $5 \%$ en el grupo con meta de hemoglobina glicosilada menor a $6 \%$; en comparación con el $4 \%$ en el grupo cuya meta era entre 7 y $7,9 \%$, RR $1,26[1,06$ a 1,51 , aumento del riesgo absoluto (ARA) 1\%, NND 100 a cuatro años para causar una muerte ${ }^{7}$. El promedio de hemoglobina $\mathrm{A} 1 \mathrm{C}$ alcanzado en los grupos de meta menor a $6 \%$ y entre 7 y $7,9 \%$ fueron 6,4 y $7,5 \%$, respectivamente.

Los demás componentes del ensayo continúan en actividad. Los pacientes asignados a control glucémico intensivo serán tratados para alcanzar las metas glucémicas mayores durante el resto del ensayo. Todos los detalles del estudio serán publicados posteriormente.

EVIDENCIA - Actualización en la Práctica Ambulatoria 


\section{¿Qué nos cuenta Steno-2 sobre las metas de glucemia?}

Steno-2 aleatorizó 160 pacientes diabéticos tipo 2 con microalbuminuria persistente a la terapia convencional o la terapia intensificada impulsada por metas, que incluye múltiples intervenciones y se centró en la modificación de la conducta ${ }^{8}$.

Una de las intervenciones en este ensayo fue la asignación a diferentes metas de hemoglobina A1C. El objetivo del tratamiento intensivo era menor a $6,5 \%$ y el del convencional fue $7,5 \%^{8}$. Los niveles promedio de hemoglobina A1C alcanzados en el grupo intensivo fueron de $7,9 \%$, en comparación con el $9 \%$ para el grupo convencional. Sin embargo, el grupo intensivo también tenía muchas otras diferentes intervenciones: objetivos de dieta, metas de ejercicio, objetivos de dejar de fumar, metas de presión arterial, objetivos de perfil lipídico indicación de uso de inhibidores de la enzima convertidora de angiotensina y un mayor uso aspirina profiláctica.

Después de ocho años de seguimiento se produjo una importante reducción en los resultados cardiovasculares y microvasculares en el grupo de intervención multifactorial y una reducción de la mortalidad no significativa, RR 0,80 [0,40 a $1,60]^{9}$. Los resultados a los 13 años de seguimiento del Steno2 recientemente publicados demuestran una reducción significativa en la mortalidad: $30 \%$ en el grupo intensivo frente a $50 \%$ en el grupo convencional, RR 0,60 [0,40 a 0,90], RRA $20 \%$, NNT 5 durante ocho años ${ }^{10}$.
Sin embargo, es imposible atribuir el beneficio sobre la mortalidad a ninguna de las múltiples intervenciones en particular $y$, en consecuencia, este ensayo no nos dice nada acerca de qué objetivo de glucemia es mejor.

\section{Conclusiones}

- Una meta de glucemia menor a $6 \%$ en comparación con una de 7 a $7,9 \%$ se asoció a un aumento de la mortalidad en diabéticos tipo 2 con alto riesgo cardiovascular.

- El objetivo óptimo de la glucemia en pacientes con diabetes tipo 2 es desconocido.

- Se necesitan ensayos clínicos adicionales que pongan a prueba los objetivos específicos de la glucemia para el espectro completo de los pacientes con diabetes tipo 2 .

$\mathrm{RR}=$ riesgo relativo

RRA = reducción del riesgo absoluto

$A R A=$ aumento de riesgo absoluto

NND = Número necesario para dañar

NNT = Número necesario a tratar

IC = Intervalo de confianza

El borrador de esta nota terpéutica fue enviada para su revisión a 40 expertos y médicos de atención primaria para co-rregir cualquier inexactitud y para asegurar que la información fuera concisa y relevante para los médicos.

\section{Referencias}

1. Canadian Diabetes Association Clinical Practice Guidelines Expert Committee. Canadian Diabetes Association clinical practice

guidelines for the prevention and management of diabetes in Canada. http://www.diabetes.ca/cpg2003 (accessed 2008 Feb 12).

2. UK Prospective Diabetes Study Group. Intensive blood glucose control with sulphonylureas or insulin compared with conventional treatment and risk of complications in patients with type 2 diabetes (UKPDS 33). Lancet. 1998;352:837-853.

3. Stratton IM, Adler Al, Andrew H, et al. Association of glycaemia with macrovascular and microvascular complications of type 2 diabetes (UKPDS 35): prospective observational study. BMJ. Aug 2000; 321:405-412.

4. Coutinho M, Gerstein HC, Wang Y, Yusuf S. The relationship between glucose and incident cardiovascular events. A metaregression analysis of published data from 20 studies of 95,783 individuals followed for 12.4 years. Diabetes Care. 22:233-240.

5. Therapeutics Initiative. Review and update 1998: Treatment of type 2 diabetes. Therapeutics Letter. \#27, Nov-Dec 1998. http://www.ti.ubc.ca/letter27 (accessed 2008 Feb 12).

6. ACCORD Study Group, Buse JB, Bigger JT, Byington RP. Action toControl Cardiovascular Risk in Diabetes trial: design and methods. Am J Cardiol. 2007;99(suppl 12A):21i-33i.

7.Action to Control Cardiovascular Risk in Diabetes (ACCORD) Trial. ACCORD Blood Sugar Treatment Strategy Announcement, February 6, 2008. http://www.nhlbi.nih.gov/health/prof/heart/other/accord/ (accessed 2008 Feb 12).

8. Gæde $\mathrm{P}$, Vedel $\mathrm{P}$, Parving HH, Pedersen $\mathrm{O}$. Intensified multifactorial intervention in patients with type 2 diabetes mellitus and microalbuminuria: the Steno type 2 randomised study. Lancet. 1999;353(9153):617-622.

9. Gaede P, Vedel P, Larsen N, Jensen GV, Parving HH, Pedersen O. Multifactorial intervention and cardiovascular disease in patients with type 2 diabetes. N Engl J Med. 2003;348(5):383-393.

10.Gæde P, Lund-Andersen H, Parving HH, Pedersen O. Effect of a multifactorial intervention on mortality in type 2 diabetes. N Engl J Med. 2008;358:580-91. 